\title{
Complete Duplication of Collecting System in a Horseshoe Kidney Presenting with Recurrent Urinary Tract Infections: Report of an Exceedingly Rare Congenital Anomaly and Review of Literature
}

\author{
Majid Mirzazadeh and Kyle A. Richards* \\ Department of Urology, Wake Forest University Baptist Medical Centre, Winston- \\ Salem, North Carolina \\ E-mail: kyrichar@wfubmc.edu
}

Received May 17, 2011; Accepted July 27, 2011; August 23, 2011

We report the fifth case in the English literature of a horseshoe kidney with a complete ureteral duplication. Our case is unique in that the previous four cases occurred in the presence of a ureterocele, whereas our patient lacked this anomaly. Further, our patient was managed conservatively, whereas the previous four patients were managed with surgery.

KEYWORDS: congenital abnormalities, urinary tract development, horseshoe kidney, duplicated collecting system

\section{INTRODUCTION}

A horseshoe kidney is the most common congenital renal fusion anomaly affecting approximately 1 in 400 live births[1]. Affected individuals may be asymptomatic. However, these kidneys are more likely than normal kidneys to have associated problems of stones and ureteropelvic junction obstruction (UPJO) that may necessitate surgical intervention.

Duplication anomalies are also relatively common, with ureteral duplication anomalies being the most common in 1 in 125 live births[2]. Ureteral duplication anomalies can be asymptomatic, but may cause symptoms of flank pain or urinary tract infection (UTI), as duplicated ureters are often associated with an obstructed upper pole moiety and a refluxing lower pole moiety. Despite the relatively frequent incidence of a horseshoe kidney and ureteral duplication anomalies in any individual patient, to the best of our knowledge, there have been just four reported cases of a horseshoe kidney and complete ureteral duplication occurring in the same patient. All four cases have had a ureterocele within the upper moiety ureter. We report our case, a 29-year-old woman with recurrent UTIs, found to have a horseshoe kidney and a complete right ureteral duplication with no ureterocele. 


\section{CASE REPORT}

A 29-year-old Caucasian female was referred to us because of recurrent UTIs. She began having febrile UTIs when she was a teenager, but did not have a thorough evaluation and was subsequently lost to follow-up. She reported an average of four febrile UTIs per year. At baseline, she denied any lower urinary tract symptoms, flank pain, or hematuria. She had a medical history of polycystic ovarian syndrome and bipolar disorder, and has not had any previous surgeries. No abnormality was found on physical exam.

Her initial evaluation included a computed tomography (CT) urogram (see Figs. 1-3), which revealed a horseshoe kidney, complete duplication of the right collecting system, marked hydroureteronephrosis of the right upper pole collecting system with severe parenchymal atrophy, and ectopic insertion of the right upper moiety ureter. She underwent examination under anesthesia, cystourethroscopy, on-table cystogram, and attempted right retrograde pyelogram. These diagnostic evaluations confirmed a completely duplicated right collecting system, with the lower pole ureteral orifice in its normal orthotopic position and the upper moiety ureteral orifice ectopically inserting into the proximal urethra without any ureterocele. Attempts to cannulate the ectopic orifice were unsuccessful due to stenosis of the ectopic orifice. An on-table cystogram was negative for vesicoureteral reflux. A Mag-3 renogram with Lasix washout (see Fig. 4) revealed decreased uptake in the expected location of the right upper pole with a split differential function of $50 \%$.

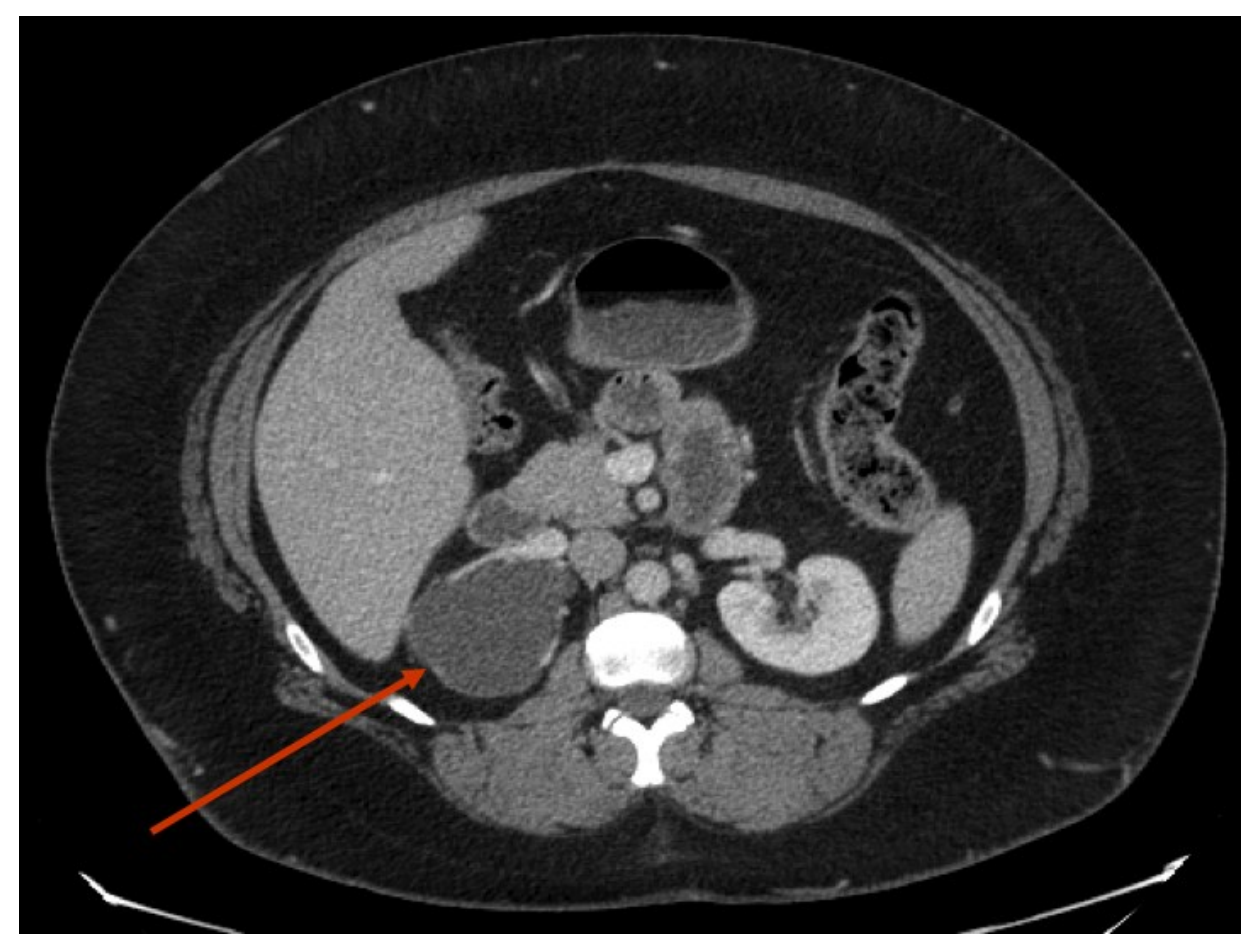

FIGURE 1. CT urogram with arrow pointing out hydronephrotic upper pole with thinned out parenchyma. 


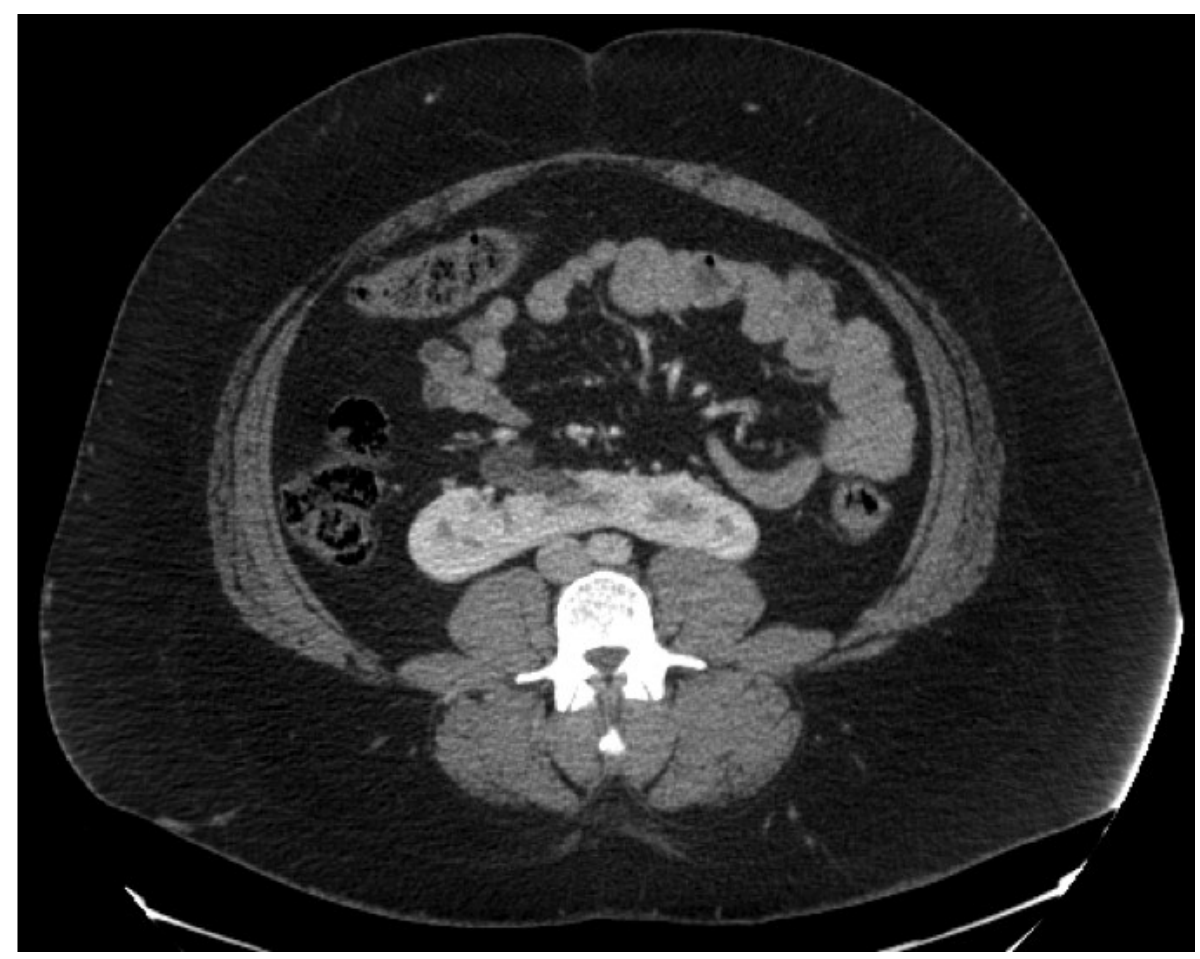

FIGURE 2. CT urogram showing isthmus of the horseshoe kidney with dilated upper pole ureter seen crossing anteriorly.

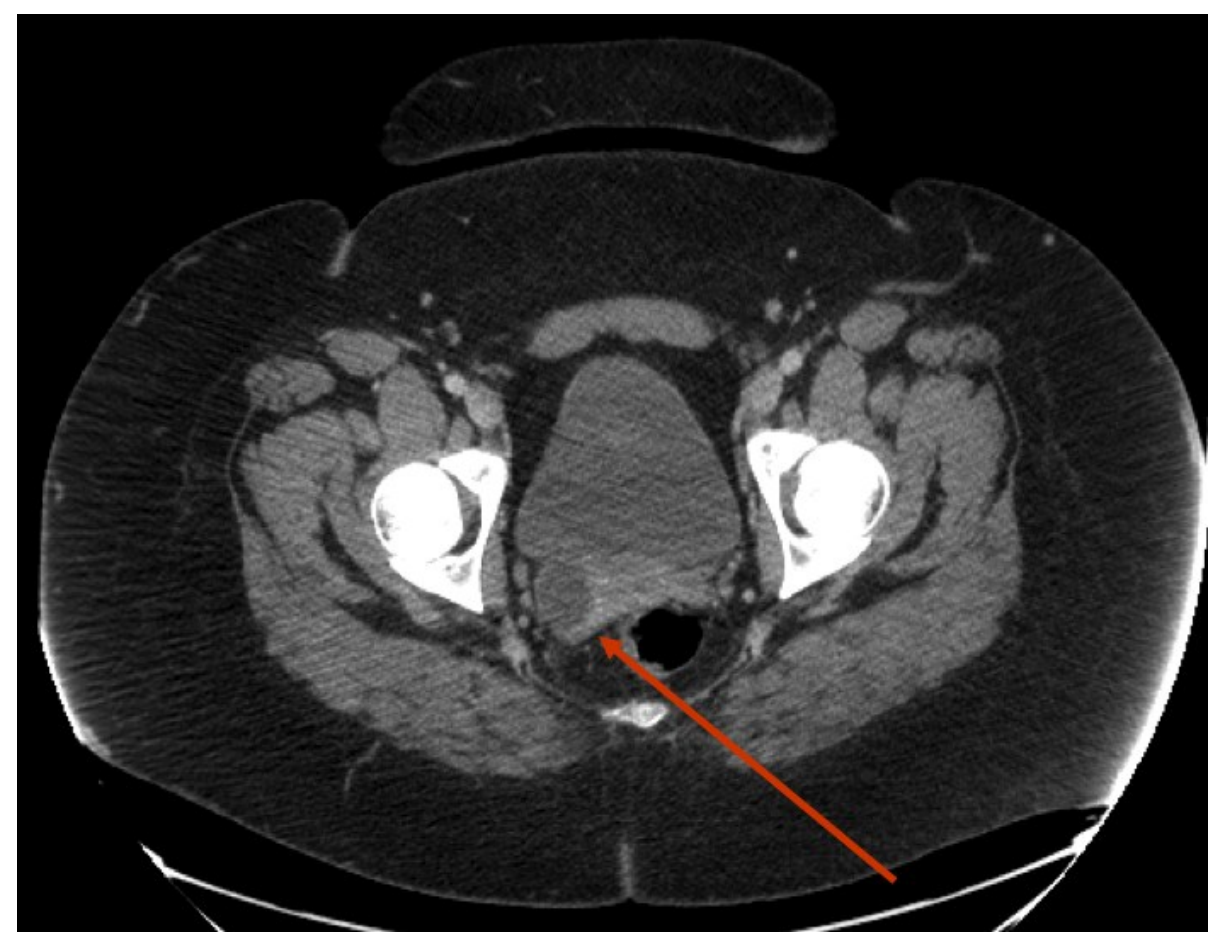

FIGURE 3. CT urogram with arrow pointing out ectopic insertion of dilated upper pole ureter. 


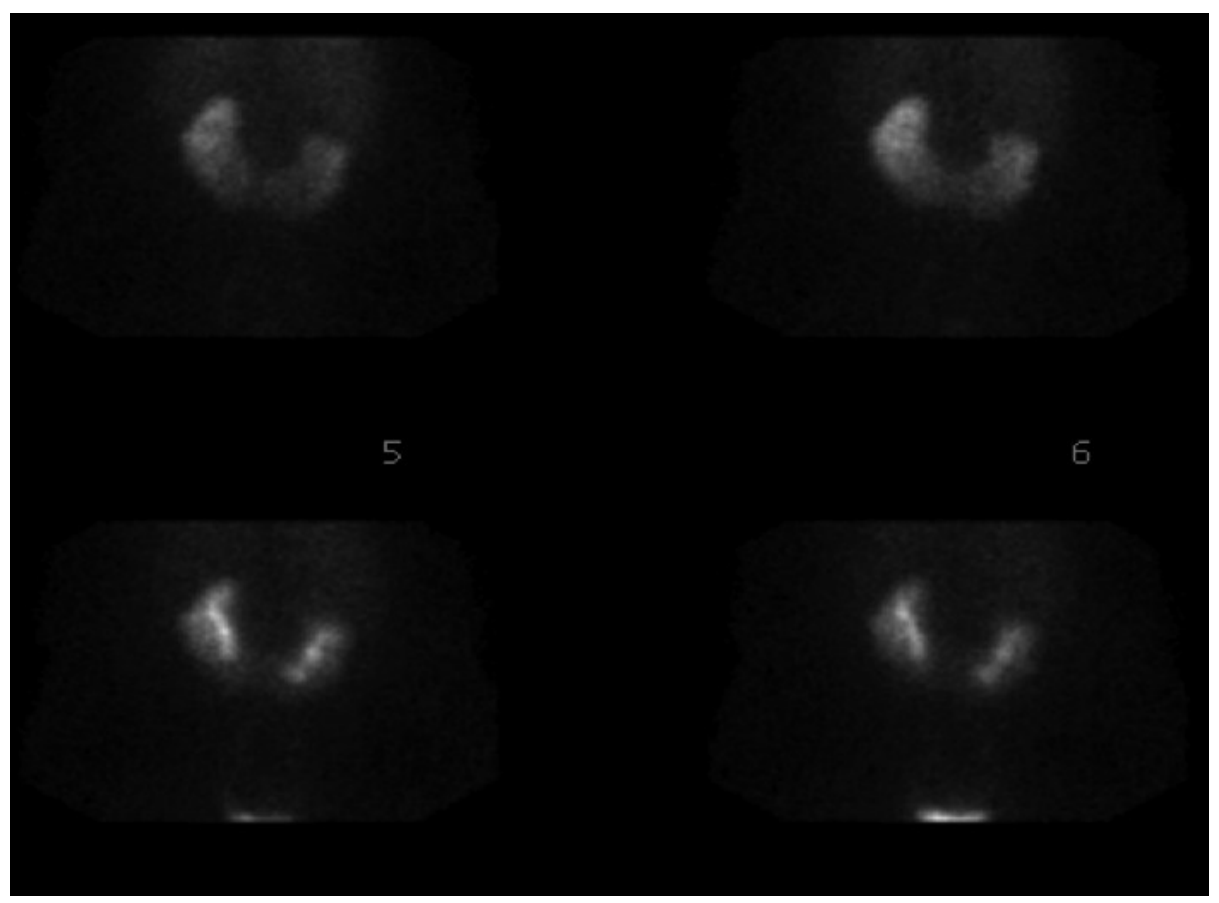

FIGURE 4. Nuclear medicine renogram showing no flow to upper pole moiety.

Treatment options including right upper pole heminephrectomy via laparoscopic, robotic, or open approaches vs. a trial of medical therapy with suppressive antibiotics was considered. Based on minimal symptoms, except recurrent UTIs, and nonfunctioning of the upper moiety of the kidney, suppressive antibiotic therapy without any surgical intervention was elected. She did not develop any UTIs on nightly nitrofurantoin for 1 year and while off the prophylaxis for 6 months.

\section{DISCUSSION AND REVIEW OF LITERATURE}

There have been four reported cases of duplex systems in horseshoe kidneys in the English literature. Segura et al. reviewed the records of 34 children with horseshoe kidneys in 1972 and identified one child with a complete ureteral duplication and a ureterocele that was managed with nephroureterectomy[3]. In 1985, Sumner and colleagues described another case involving a 3-year-old boy that presented with urinary retention[4]. He was found to have a horseshoe kidney and a complete left ureteral duplication associated with a left ureterocele that was successfully managed with a left upper pole heminephrectomy. A third case was reported by Mikuma and associates involving a 55-year-old man who was managed via upper pole ureteropyelostomy with ligation of the distal ureter and unroofing of the ureterocele[5]. Lastly, in 2009, Chiang and Kaplan reported a 2-month-old full-term female with febrile UTI despite antibiotic prophylaxis that was found to have a left ectopic ureterocele[6]. Their attempts at endoscopic incision were unsuccessful and a nephrostomy tube was placed. A dimercaptosuccinic acid (DMSA) scan was performed, and demonstrated the presence of an isthmus and minimal left upper pole function. Further anatomy was delineated using a CT scan with visualization of a horseshoe kidney and the ureterocele. A left upper pole partial nephrectomy with open ureterocele marsupialization and lower pole reimplantation was performed.

All of the four reported cases had common features, including a ureterocele in the distal end of the upper pole ureter. Only one of the cases was an adult, and the other three involved children. There does not seem to be a predilection for sex, as the frequency has been 1:1 male to female in the four reported 
cases in the literature. All cases were treated surgically. To the best of our knowledge, our case is the fifth case reported in literature and the first horseshoe kidney with a duplex system without a ureterocele. It is also unique as the only one managed conservatively.

While the fusion anomalies and duplication anomalies frequently occur independently, their occurrence together seems to be a truly rare event. Embryologically, completely duplicated systems arise as two separate ureteric buds originating from the mesonephric duct to interact with the metanephric blastema. Classically, Meyer noted that the upper pole ureteral orifice is consistently distal or lower in relationship to the lower pole ureteral orifice, which is located more cranially or higher up in the bladder[7]. The upper pole ureteral orifice is often ectopic and can be located in a variety of locations, including the proximal urethra, as was seen in our patient.

A horseshoe kidney, on the other hand, is formed between the $4^{\text {th }}$ and $6^{\text {th }}$ week of gestation after the ureteric bud has entered the renal blastema. Because of one of the several proposed mechanisms, the lower poles of the kidneys fuse, forming an isthmus of connective or functional tissue. This happens before the kidneys have ascended or rotated on their long axis. The inferior mesenteric artery prevents the kidneys from ascending to their usual place in the upper abdomen by obstructing the movement of the isthmus. A horseshoe kidney is frequently found to be associated with other congenital anomalies. One study identified associated anomalies in up to one-third of 96 patients in the study, but none of which were ureteral duplications[8].

Management of patients with duplicated collecting systems and/or horseshoe kidneys is largely dependent on the symptoms and clinical presentations. Our particular patient of interest was found to have a poorly functioning right upper pole moiety in a completely duplicated collecting system in a horseshoe kidney when she presented with recurrent UTIs. Zumsteg and colleagues recently reported their experience at the University of Michigan with laparoscopic heminephrectomy for benign renal anomalies, including eight patients with ureteral duplication, two with horseshoe kidneys, and one with crossed-renal ectopia[9]. All surgeries were completed laparoscopically with minimal blood loss. Renal function was preserved in all patients and all symptoms resolved in the patients postheminephrectomy. Laparoscopic upper pole heminephrectomy was also described by Abouassaly and coworkers in five patients with duplex collecting systems[10]. In this series, one patient developed a urinoma. Additionally, authors from Iran reported on the successful use of bipolar energy to transect the isthmus of a horseshoe kidney in three patients undergoing laparoscopic heminephrectomy for benign disease[11]. Researchers from Long Island Jewish Medical Center found the laparoscopic endostapler to be a useful adjunct for division of the isthmus during laparoscopic heminephrectomy in the horseshoe kidney[12]. While laparoscopic heminephrectomy for the management of benign disease in horseshoe kidneys appears to be feasible, challenges do exist owing to the abnormal vasculature and aberrant anatomy of these kidneys. Our patient has been off suppressive antibiotic prophylaxis for 6 months, now being 18 months without any breakthrough UTIs or any decline in her renal function, with no surgical intervention.

\section{REFERENCES}

1. Bauer, S.B. (2007) Anomalies of the upper urinary tract. In Campbell-Walsh Urology. 9th ed. Wein, A.J., Kavoussi, L.R., Novick, A.C., Partin, A.W., and Peters, C.A., Eds. Saunders Elsevier, Philadelphia. p. 3283.

2. Schlussel, R.N. and Retik, A.B. (2007) Ectopic ureter, ureterocele and other anomalies of the ureter. In CampbellWalsh Urology. 9th ed. Wein, A.J., Kavoussi, L.R., Novick, A.C., Partin, A.W., and Peters, C.A., Eds. Saunders Elsevier, Philadelphia. p. 3413.

3. Segura, J.W., Kelalis, P.P., and Burke, E.C. (1972) Horseshoe kidney in children. J. Urol. 108, 333-336.

4. Sumner, T.E., Volberg, F.M., Munitz, A., Harrison, L.H., and Mashburn, A.M. (1985) Unilateral duplex horseshoe kidney with ectopic ureterocele. South. Med. J. 78, 199-201.

5. Mikuma, N., Adachi, H., Takatsuka, K., Yokoo, A., Wada, H., and Takahashi, A. (1996) Ectopic ureterocele with a horseshoe kidney in an adult. Int. J. Urol. 3, 243-244.

6. Chiang, G. and Kaplan, G. (2009) Management of ectopic ureterocele with horseshoe kidney. J. Pediatr. Urol. 5, 400-401.

7. Meyer, R. (1946) Development of the ureter in the human embryo: a mechanistic consideration. Anat. Rec. 96, 355. 
8. $\quad$ Boatman, D.L., Culp, D.A., Jr., Culp, D.A., and Flocks, R.H. (1972) Crossed renal ectopia. J. Urol. $108,30$.

9. Zumsteg, J., Roberts, W.W., and Wolf, J.S. (2010) Laparoscopic heminephrectomy for benign renal anomalies. J. Endourol. 24(1), 41-47.

10. Abouassaly, R., Gill, I.S., and Kaouk, J.H. (2007) Laparoscopic upper pole partial nephrectomy for duplicated renal collecting systems in adult patients. Urology 69, 1202-1205.

11. Nouria-Mahdavi, K. and Izadpanahi, M.H. (2008) Laparoscopic heminephrectomy in horseshoe kidney using bipolar energy: report of 3 cases. J. Endourol. 22(4), 667-669.

12. Yohannes, P., Dinlenc, C., Liatsikos, E., et al. (2002) Laparoscopic heminephrectomy for benign disease of the horseshoe kidney. JSLS 6, 381-384.

\section{This article should be cited as follows:}

Mirzazadeh, M. and Richards, K.A. (2011) Complete duplication of collecting system in a horseshoe kidney presenting with recurrent urinary tract infections: report of an exceedingly rare congenital anomaly and review of literature. TheScientificWorldJOURNAL: TSW Urology 11, 1591-1596. DOI 10.1100/tsw.2011.143. 


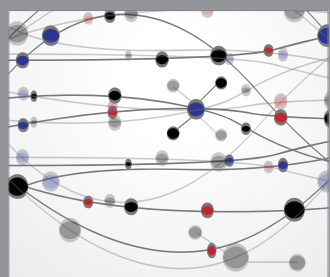

The Scientific World Journal
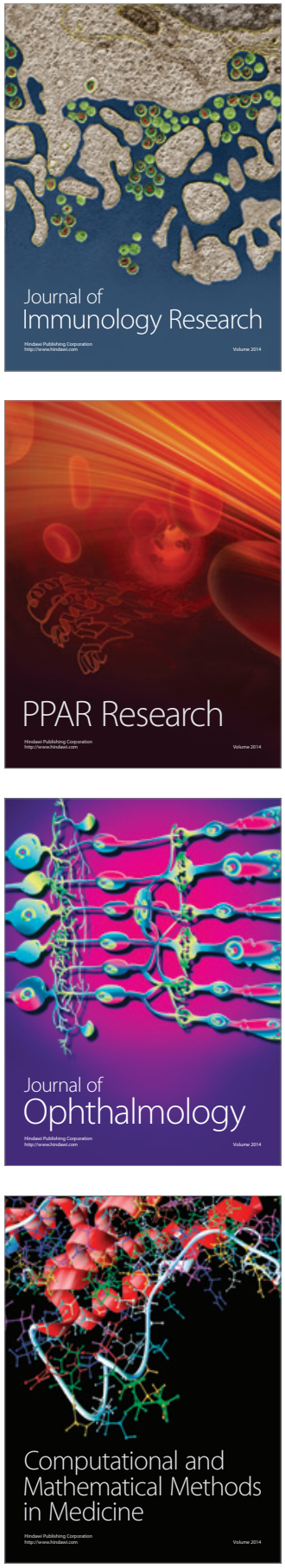

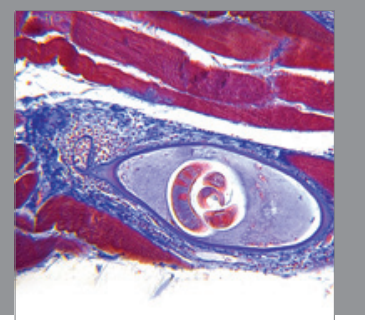

Gastroenterology

Research and Practice
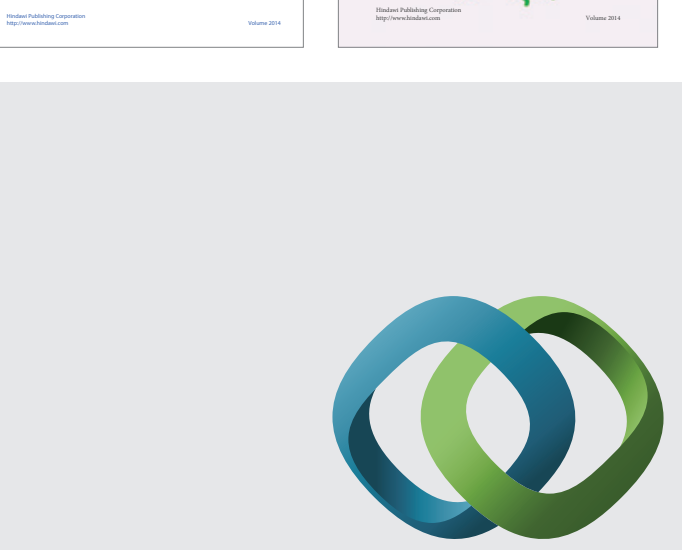

\section{Hindawi}

Submit your manuscripts at

http://www.hindawi.com
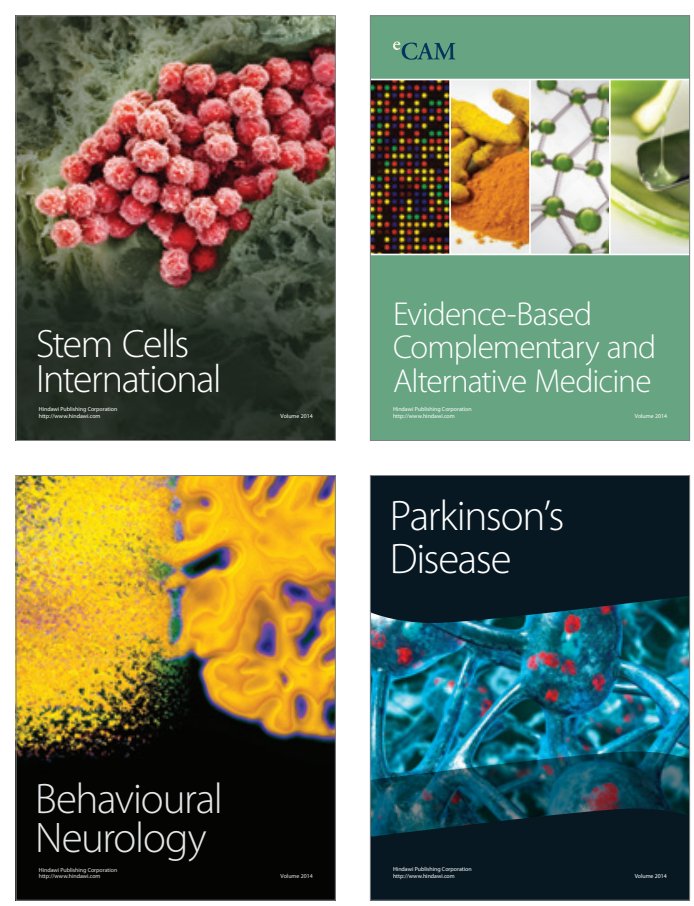

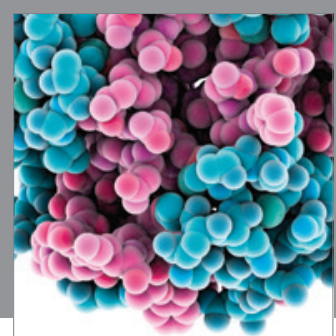

Journal of
Diabetes Research

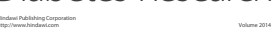

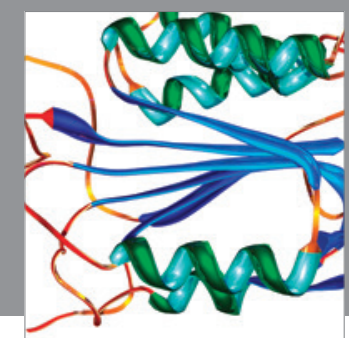

Disease Markers
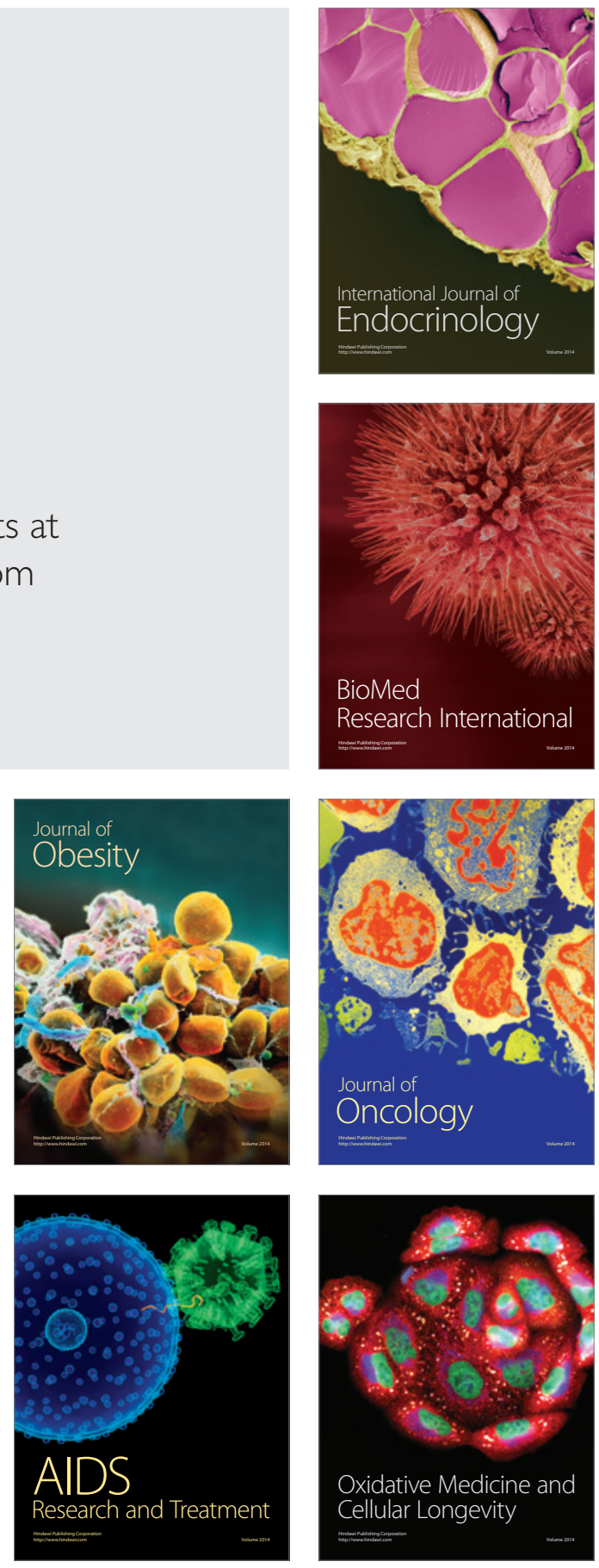